\title{
Driver Safe Speed Model Based on BP Neural Network for Rural Curved Roads
}

\author{
Xiaolei Chen ${ }^{1}$, Ruijuan $\mathrm{Chi}^{1}$, Jianqiang Wang ${ }^{2}$, Changle Pang ${ }^{1, *}$, and Qing Xiao ${ }^{1}$ \\ ${ }^{1}$ College of Engineering, China Agricultural University, \\ Tsinghua East Road No.17, Beijing, 100083 \\ pangcl@cau.edu.cn \\ ${ }^{2}$ State Key Laboratory of Automotive Safety and Energy, Tsinghua University, \\ Shuangqing Road No.30, Beijing, 100084
}

\begin{abstract}
In order to improve the safety and comfort of the vehicles on rural curved roads, the paper proposed a safe curve speed model based on the BP Neural Network. A series of drivers' manual operation state data during cornering were gathered and observed according to the driver experiments under real traffic conditions. Three factors, referring to the speed calculated based on road trajectory parameters, the adhesion workload and the yaw rate computed from the processed data, were used as inputs of the model to obtain the target vehicle speed. Finally, tests verify the applicability of the modified model. It indicates that the developed speed model can adjust to the individual curve speed behavior of each driver.
\end{abstract}

Keywords: Rural curved road, driver behavior, adhesion workload, yaw rate, BP Neural Network.

\section{Introduction}

Curves of the rural roads are those places where the accidents take place more frequently than other parts of the road due to their intricate road condition. Recently in China, statistics on traffic accidents from the Chinese Ministry of Transportation in 2009 showed that 25,146 road accidents occurred on curved road, which is up to $10.56 \%$ of the total accidents [1]. Besides, the occurrence of these accidents is increasing. Mistakes during steering is one of the main factors, which fundamentally results from the driver's incorrect recognition or misjudgment of road condition, vehicle performance or surrounding environment, that normally let the driver enter a corner at a high speed [2].

In order to solve the aforementioned problems, many studies have been conducted to calculate the safe speed for cornering and several models have been developed during the last few decades [3-8]. According to [3], a predicting model of the 85th percentile speed was developed by Krammers in 1993, and Polus built four speed models based on numbers of speed data to estimate the operating speed for the twolane rural roads. Another curve safe speed model which was used in accident

\footnotetext{
* Corresponding author.
} 
reconstructions was developed in [5].Taking the acceptable lateral acceleration into account, a safe speed was presented by the Spanish Road Administration [7]. It was found in [8] that a differential speed model of the 85th percentile speed and the operating speed had been suggested for the two-lane rural roads according to the experiment data. In general, all the speed models above had determined the safe speed according to the road trajectory, yet considering the driver's individual characteristic. Sometimes it would make the drivers feel uncomfortable when cornering. As a result, the curve safe speed model should take into account of the individual characteristic of each driver.

Meanwhile, some researches had been undertaken focusing on the driver behavior on curved roads in recent years [9-13]. According to [9], the lateral force coefficient is a very important parameter to the safety and comfort of driving. It was indicated in [12] that drivers chose their comfortable speed mainly depending on curvature and driving conditions. Besides, the comfort of driving was bound up with the value of lateral acceleration. However, the common objective of existing researches regarding driver behavior on curved roads is to meet the requirement of geometric design of highways. Therefore existing models may be unsuitable to improve the safety and comfort on curved roads.

In this study, a driver safe speed model on curved roads is developed based on real experimental data gathered from a series of driver behavior experiments. With a view of adapting to driver's individual curve speed behavior, the Neural Network method is used in the driver safe speed model. Tests verify the applicability of the modified model.

\section{Driver Behavior Experiments}

\subsection{Data Acquisition System}

As shown in Table 1, three types of data, which could reflect driver behavior and vehicle states, were recorded with the data acquisition system.

Table 1. Data collected based on real traffic experiments

\begin{tabular}{ll}
\hline Type & Variables \\
\hline \multirow{3}{*}{ Vehicle states } & Vehicle speed \\
& Yaw rate \\
& Lateral acceleration \\
& Longitudinal acceleration \\
& Relative speed \\
\cline { 2 - 2 } Driver operation & Steering wheel angle \\
& Brake pedal depression \\
& Gas pedal depression \\
\cline { 2 - 2 } Road information & GPS coordinates \\
& Road conditions \\
\hline
\end{tabular}


The data were collected from CAN bus of the instrumented vehicle and some appendix sensors. Besides, the curvature and the friction coefficient of the road could be estimated in accordance with the GPS data and the vehicle states.
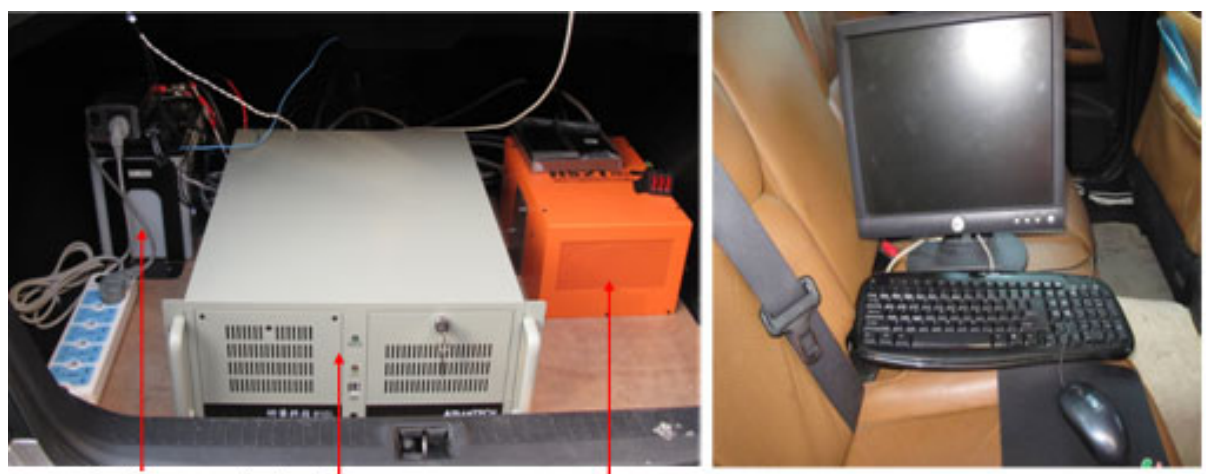

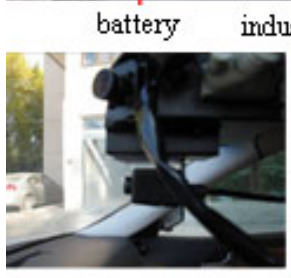

Front View Camera

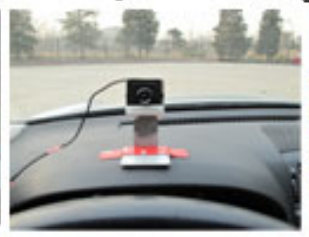

Driver Face Camera

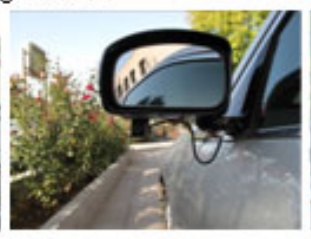

Left Rear View Camera

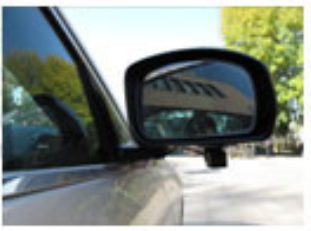

Right Rear View Carnera

Fig. 1. Data acquisition system

As indicated in Fig. 1, the data acquisition system consists of a battery, an industrial computer, and a driving recorder which were installed in the car boot. A monitor was fixed on the back of the co-pilot seat for the experiment recorder. In addition, four cameras were fixed in the system to record different images in real time. The data collection frequency of this system was $30 \mathrm{~Hz}$.

\subsection{Experiment Route and Design}

Considering the requirement and purpose of the experiments, the sample route was required to be roads with regular curves. So we chose a section of a highway that started from the south entrance of the Beijing-Tibet highway to Badaling as the experiment area, as shown in Fig. 2.

A sample of 18 participants with full China driving license was enrolled to accomplish the experiments from Beijing. These drivers were acquainted with the road conditions and were required to drive with a safe and comfortable speed in free traffic flow. Data were collected and saved with the data collection system. It should be noted that only part of each experiment data from the curve entry to the curve exit were used for the analysis. 


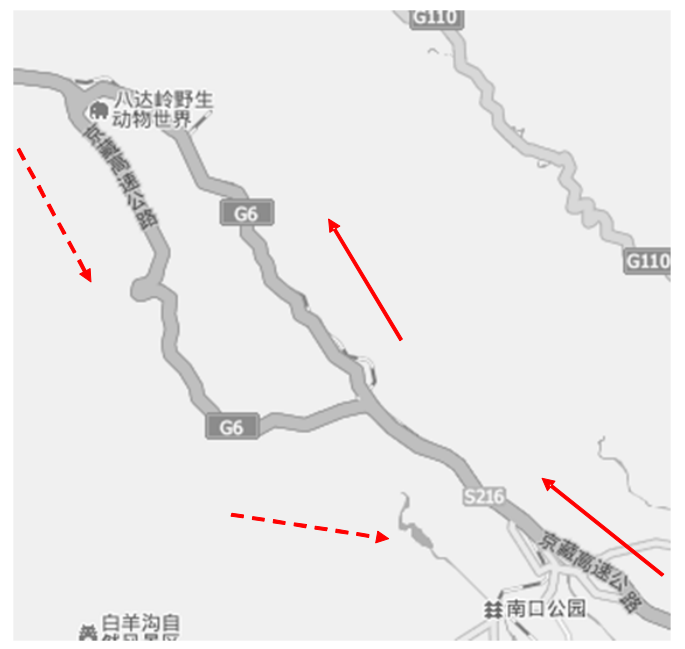

Fig. 2. Experiment route

\section{Data Observation}

Direct data could not satisfy the requirements of the model due to error and noise. The observation of the original data, such as longitudinal acceleration, lateral acceleration, and yaw rate, was taken place in the next section.

\subsection{Kalman Filter of the Original Data}

The Kalman Filter is a set of mathematical equations that provides an efficient computational (recursive) means to estimate the state of a process, in a way that minimizes the mean of the squared error [14]. It is widely used in many fields because of its powerful features.

Assume that the original data is $x_{k}$, and develop it by the formula of Tailor in time domain. The state equation is shown in Eq.1 taking $x_{k}$ and $x_{k}^{\prime}$ as state variables.

$$
X_{k}=\varphi X_{k-1}+A w_{k}
$$

Where, $X_{k}=\left[x_{k}, x_{k}^{\prime}\right]^{T}, \varphi=\left[\begin{array}{cc}1 & \Delta T \\ 0 & 1\end{array}\right], \mathrm{A}=\left[\begin{array}{c}\frac{\Delta T^{2}}{2} \\ \Delta T\end{array}\right], w_{k}=x_{k}^{\prime \prime}$ is the random noise, $\Delta T$ is the sampling time, and $\Delta T=1 / 30 \mathrm{~s}$.

The measurement equation is shown in Eq.2.

$$
z_{k}=H X_{k-1}+n_{k}
$$


Where, $z_{k}$ is the measurement value of the data, $H=\left[\begin{array}{ll}1 & 0\end{array}\right]$, and $n_{k}$ is the measurement noise.

The optimal estimation of the parameters based on Kalman Filter is shown in Eq.3.

$$
\hat{X}_{k}=\hat{X}_{k}^{-}+K_{k}\left(z_{k}-H_{k} \hat{X}_{k}^{-}\right)
$$

Where, $\hat{X}_{k}$ is the optimum estimate of $X_{k}, \hat{X}_{k}^{-}$is the priori estimate of $X_{k}$, $K_{k}$ is the Kalman gain, and $z_{k}$ is the measurement vectors.

The recursive process of the system is as follows:

$$
\begin{gathered}
\hat{X}_{k}^{-}=\varphi \hat{X}_{k-1}^{-} \\
P_{k}^{-}=\varphi P_{k-1} \varphi^{T}+A Q_{k} A^{T} \\
K_{k}=P_{k}^{-} H_{k}^{T}\left(H_{k} P_{k}^{-} H_{k}^{T}+R_{k}\right)^{-1} \\
P_{k}=\left(1-K_{k} H_{k}\right) P_{k}^{-}
\end{gathered}
$$

Where, $Q_{k}$ is the process noise covariance, $R_{k}$ is the measurement noise covariance. $P_{k}, P_{k}^{-}$are the optimum estimate error covariance and the posteriori estimate error covariance, respectively. Their initial values are set to be zero matrixes.

The comparison result of data before and after filtering is shown in Fig. 3 taking longitudinal acceleration as example.

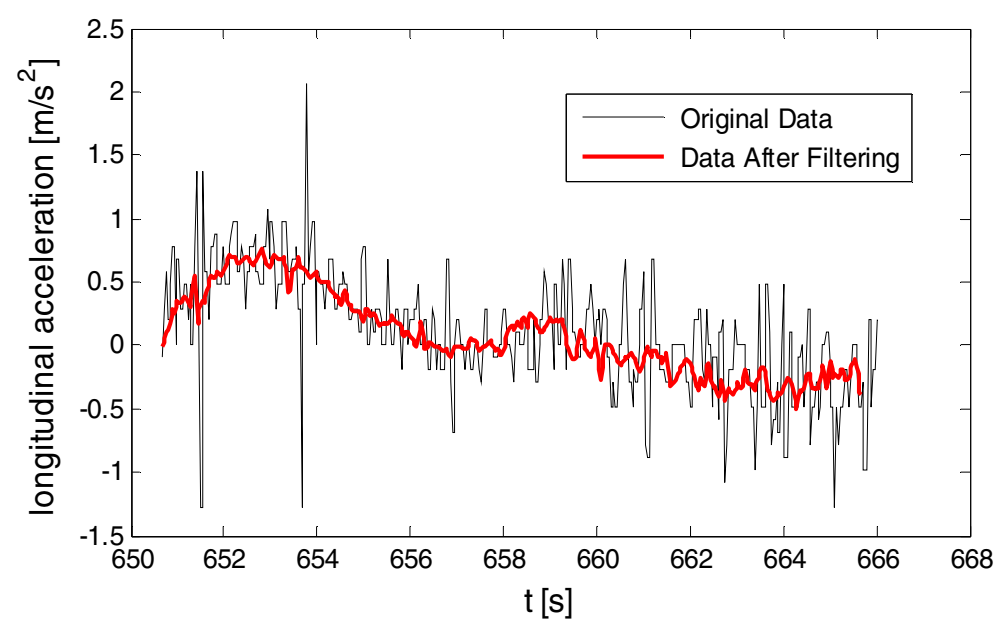

Fig. 3. Comparison of the data before and after filtering 


\subsection{Error Compensation of the Original Data}

In order to reflect the real states of the vehicle, the deviation compensation between the measurement data and the real values caused by installation error, the roll and the pitch of the vehicle is extremely essential.

The influence of the roll and pitch of the vehicle to the parameters is shown in Fig. 4. The approximation of the roll angle can be estimated with the model [15].
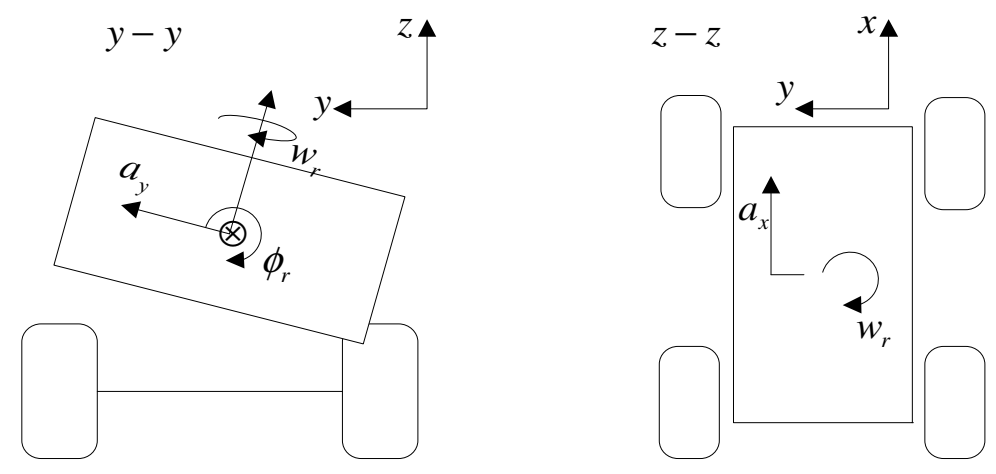

Fig. 4. Influence of the roll and pitch of vehicle to data

As shown in Eq.8, a steady -state moment equation is determined from Fig. 4 based on the static suspension model.

$$
\left(K_{\phi_{r} f}+K_{\phi_{r} r}\right) \phi_{r}=m a_{y s} h^{\prime} \cos \phi_{r}-m g h^{\prime} \sin \phi_{r}
$$

Where, $K_{\phi_{r} f}, K_{\phi_{r} r}$ are the roll angle rigidity of the suspension, $\phi_{r}$ is the roll angle of the vehicle, $a_{y s}$ is the measurement lateral acceleration, and $h^{\prime}$ is the distance between mass center and roll center.

It can be considered that $\cos \phi_{r} \approx 1$ and $\sin \phi_{r} \approx 0$ when the value of the roll angle is small. Eq. 8 can be converted to the form as Eq.9.

$$
\phi_{r}=\frac{m a_{y s} h^{\prime}}{K_{\phi_{r} f}+K_{\phi_{r} r}-m g h^{\prime}}
$$

The actual values of the parameters can be calculated with Eq.10, Eq.11 and Eq.12.

$$
\begin{gathered}
a_{y}=\frac{a_{y s}+g \sin \phi_{r}}{\cos \phi_{r}} \\
w_{r}=\frac{w_{r s}}{\cos \phi_{r}}
\end{gathered}
$$




$$
a_{x}=a_{x s}-l_{y} w_{r}^{\prime}
$$

Where, $w_{r}$ is the measurement yaw rate, and $a_{x s}$ is the measurement longitudinal acceleration. $l_{y}$ is the distance between mass center and installation position.

The data which had been compensated are more close to the actual values, and can be used for practical application.

\section{Safe Curve Speed Model Based on BP Neural Network}

BP Neural Network, which has flexible topology structure and self-adaptive capacity and has redundant organized fashion and perseverance, is widely used for the nonlinear system in many fields [16].

Drivers as a complex nonlinear system adjust their appropriate speed according to many effect factors, such as driving conditions, their own states when cornering etc. In this study, a curve speed model which can adapt to the driver's individual characteristic is established based on the BP Neural Network because of its superiority on intricate system. As shown in Fig. 5, three variables are taken as input data. A two layers feed-forward BP Neural Network is used to simulate the motion state of the vehicle on curved roads. The output data of the model is the target vehicle speed.

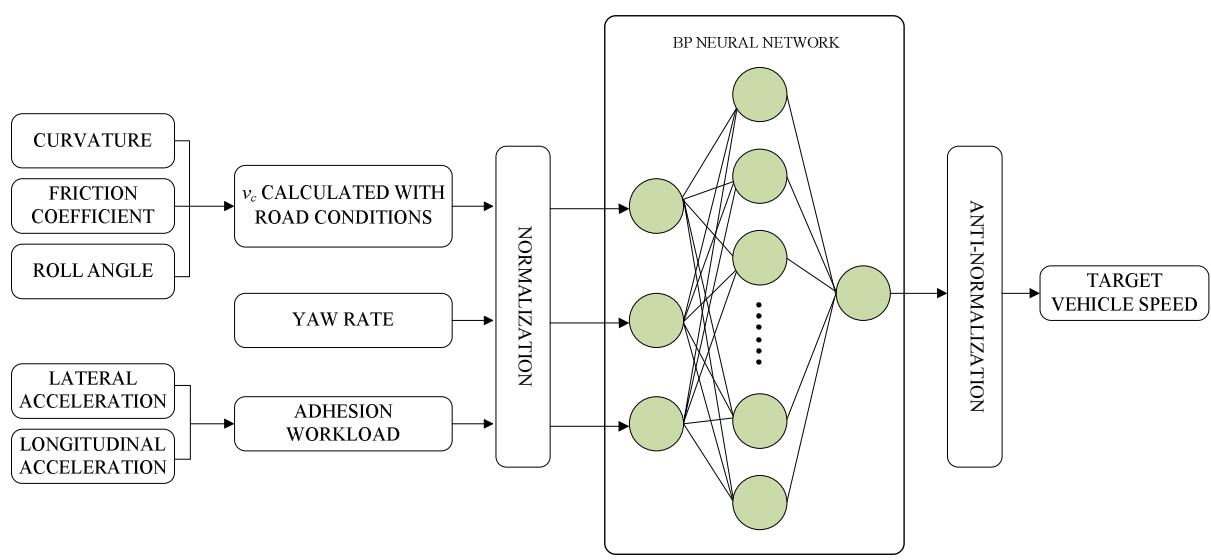

Fig. 5. Driver safe speed model based on BP Neural Network

There are three factors taken as inputs of the BP Neural Network. The first one is the speed calculated considering road trajectory. As shown in Eq.13, three parameters are chosen to describe the curved road conditions. 


$$
v_{c}=\sqrt{\frac{g}{c}\left(\frac{\phi+\mu}{1-\phi \mu}\right)}
$$

Where, $v_{c}$ is the safe speed on curved road, $c$ is the curvature, $\phi$ is the road superelevation angle, and $\mu$ is the friction coefficient.

The second one is the yaw rate collected from the real road experiments. The last one is the adhesion workload which can reflect the ratio of the friction coefficient used by drivers. It is varied with different characteristics of the drivers, and the calculative method of it is shown in Eq.14.

$$
\eta=\sqrt{\frac{a_{x}^{2}+a_{y}^{2}}{\mu_{\max } g}}
$$

Where, $\eta$ is the adhesion workload, $a_{x}$ is the longitudinal acceleration, $a_{y}$ is the lateral acceleration, and $\mu_{\max }$ is the maximum friction coefficient.

Firstly, calculate the values of the speed, adhesion workload, and the yaw rate based on a fifty seconds length of data chosen from the abovementioned experiments. Then, normalize the input data and the output data into the range $0-1$, and take them as the training set to train the networks. After that, set up the parameters of the networks. The number of the hidden layer nodes is set to 60 considering the complexity of the driver's characteristic. The transfer functions of the hidden layer and the output layer are "logsig" and "pureline", respectively. The root mean square error value used for the stopping signal was set to 0.001 , and the number of epochs was set to 100. All the iterations realize the target mean square error in less then 7 epochs. The output data could be converted to the target speed based on antinormalization.

\section{$5 \quad$ Evaluation and Discussion}

\subsection{Evaluation}

The evaluation was carried out based on the data gathered from real road experiments. Firstly, several parameters, such as speed $\left(v_{c}\right)$, adhesion workload, yaw rate etc was calculated or processed according to the actual data of the drivers on curved roads. Secondly, train the Neural Networks with the information above to make it adapt to the driver's characteristic. Finally, when the vehicle driving into the curve, the model began to work and provided a safe and comfortable speed for the driver.

The comparison result of the real speed and the target speed known as the output of the model is shown in Fig. 6.

As shown in the figure, the model can reflect the curve speed behavior of the drivers, and provide a safe and comfortable speed when turning. 

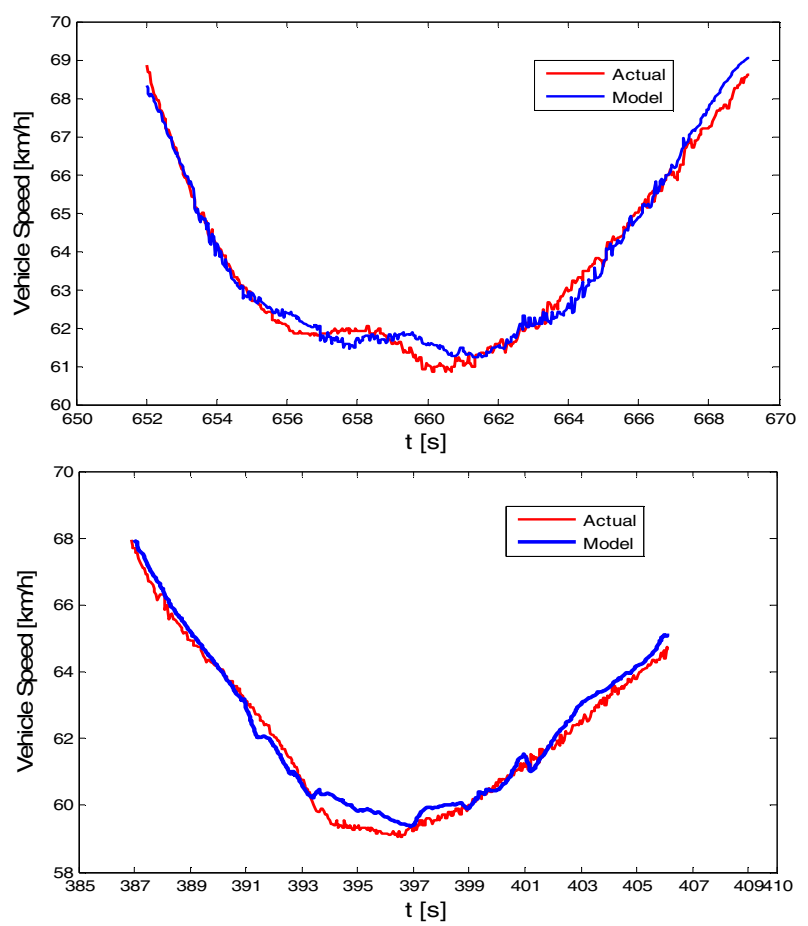

Fig. 6. Validation of driver safe speed model (No.11 and No.12 drivers)

\subsection{Discussion}

Two factors referring to the road trajectory (e.g. curvature, the road superelevation angle, and the friction coefficient) and the characteristic of driver (e.g. the adhesion workload and the yaw rate) were considered in the curve speed model in this paper. However, there are several parameters that had not been taken into account, including the visibility, the slope angle of the road and the initial velocity etc. Besides, the driver behavior experiments were carried out with no vehicles ahead, the driver's characteristic might change when following other cars. A further study with accordance into these factors will be done in the next reach.

In addition, some of the drivers participated in the experiments didn't obey the rules strictly we had made earlier, which might impact the data validity. Besides, the sample size of the experiments also has influence on the result. It is assumed that the result would be improved by increasing the amount of the experimental data.

\section{Conclusion}

This paper focused on a driver safe speed model on rural curved roads by considering the driver's curve speed characteristic. From what had been discussed above, the conclusions could be drawn as follows: 
(1) The developed safe speed model is proposed on account of the actual driver behavior experimental data, and is able to provide a more suitable speed for drivers when cornering.

(2) The processed parameters taken as the inputs of the model can reflect the real conditions of the rural curved roads and the actual curve speed behavior of the drivers.

(3) The driver safe speed model based on BP Neural Network is adaptive to the individual curve speed characteristic of each driver. It can improve the safety and comfort of the vehicle on curved roads.

Acknowledgements. The research reported herein was supported by the Chinese National Programs for High Technology Research and Development, No. 2011AA110402. The authors would like to thank Dezhao, Zhang (Tsinghua University) for his kind support for the paper preparation.

\section{References}

1. Statistical Yearbook on Road Traffic Accidents of China (2008). Ministry of Transportation of China (2009)

2. Lee, Y.H., Deng, W.: Speed Control Method for Vehicle Approaching and Travelling on a Curve. US Paten, No.0150157 (2007)

3. Stamatiadis, N., Gong, H.: Analysis of Inconsistencies Related to Design Speed, Operating Speed and Speed Limits. University of Kentucky College of Engineering (2007)

4. Pomerleau, D., Jochem, T., Thorpe, C.: Run-off-road Collision Avoidance Using IVHS Countermeasures. National Highway Traffic Safety Administration (1999)

5. Sledge, N.H., Marshek, K.M.: Formulas for Estimating Vehicle Critical Speed from Yaw Marks. A Review, SAE (1997)

6. Zhong, X., Ren, F., Rong, J.: The Two-step Model of Operating Speed on Horizontal Curves Based on Driver's Information Collection and Processing. Transportation Engineering, 307-312 (2004)

7. Mopu.: Seńalización Vertical. Instrucción Decarreteras. Norma 8.1-IC. Centro de Publicaciones Del Ministerio de Fomento (2000) (in Spanish)

8. Misaghi, P., Hassan, Y., Asce, M.: Modeling Operating Speed and Speed Differential on Two- Lane Rural Roads. Journal of Transportation Engineering, 408-417 (2005)

9. Zheng, K., Rong, J., Ren, F.: Analysis of Relation between Curve's Radius and Steering Speed on Expressway. Journal of Highway and Transportation Research and Development 20(2), 28-30 (2003)

10. Abdelwahab, W.M., Aboul-Ela, M.T., Morrall, J.F.: Geometric Design Consistency Based on Speed Change on Horizontal Curves. Road Transport Res. 127(1), 13-23 (1998)

11. Zhang, K., Jiang, L., Rong, J., Ren, F.: Study on Mental and Physiological Effects of Horizontal Radius of Expressway on Driving Reaction. Journal of Highway and Transportation Research and Development 21(5), 5-7 (2004)

12. Canadian Researchers Test Driver Response to Horizontal Curves. Journal of Road Management and Engineering (1998)

13. McFadden, J., Elefteriadou, L.: Evaluating Horizontal Alignment Design Consistency of Two-lane Rural Highways: Development of New Procedure. Transportation Research Record, 1737, 9-17. Transportation Research Board, Washington, D.C (2000) 
102 X. Chen et al.

14. Welch, G., Bishop, G.: An Introduction to the Kalman Filter. Department of Computer Science University of North Carolina at Chapel Hill (2006)

15. Li, L.: Cooperative Control of Vehicle Stability Based on AFS and DYC, Doctoral Thesis of Tsinghua University (2009)

16. Wei, L., Yumin, S.: Prediction of Energy Production and Energy Consumption Based on BP Neural Networks. In: Proceedings of the IEEE International Symposium, pp. 176-179. IEEE Press (2008) 